\title{
A Study Of Acute Myocardial Infarction In Pregnancy And Puerperium
}

\author{
${ }^{1}$ Dr Adilakshmi .B, Dr Darga saheb peer Dm \\ M D DM Associate professor of cardiology, siddhardha medical college, vijayawada,AP,India
}

\section{Introduction}

To estimate the population-based incidence and to study. Pregnency and MI effects Patients were divided into 4 group: antenatal diagnosis, intrapartum diagnosis, up to 6-week postpartum diagnosis, and those without the diagnosis of acute MI. All groups were compared by student $t$ test or $X^{2}$ or both, where appropriate.

Results : A total of 30 women had an acute MI during the antepartum (38\%) intrapartum (21\%), or 6week postpartum (41\%) period, The maternal mortality rate was $7.3 \%$, and maternal death only occurred in women with an acute MI before or at delivery $(\mathrm{P}<.01)$. Compared with women who did not have an acute MI, those with one were more likely to be older (30\% were older than 35 years compared with $10 \%)$, multiparous (78\% compared with $61 \%$ )

(ML Multivariate analysis identified chronic hypertension, diabetes, advancing maternal age, edampsia, and severe preedampsia as independent risk factors for acute ML.

Acute myocardial infarction (MI) during pregnancy and the puerperium is a rare but often catastrophic event, associated with significant maternal mortality. ${ }^{1.2}$ In prior meta-analysis of case reports, maternal mortality has been estimated to be from $19 \%$ to as high as $37 \%$. 2 In addition, it seems that maternal mortality is higher if it occurs in the third trimester or postpartum period. 2-4 Neonatal mortality, on the other hand, has been reported to be less than that of the mother but still quite elevated (13\% to 17\%) when compared with the population at large.

The incidence of acute MI during pregnancy has been determined to occur with a frequency of 1 in 10,000 to 1 in 30,000 preganancies. . $^{1-3 .}$

Maternal morbidity and other preganancy outcomes of acute MI during pregnancy have been previously reported to be increased, but not often clearly defined. Several reports have examined the associations of cardiac risk factors and women suffering an acute MI during pregnancy. In one study, cardiac risk factors were found in 58\% of pregnant women with acute MI, suggesting that these high-risk patients may be identifiable. 1 Of those risk factors reported, hypertension and smoking were most prevalent and found in $25 \%$ and $20 \%$ of cases respectively. 1 Diabetes mellitus (not identified as gestational or pregestational) was identified in 3-5\% of these patients. Hypercholesterolemia, hyperlipidemia, and obesity were all found in less than $5 \%$ of cases reported, and intuition would suggest that obesity alone should be more common than that reported in the normal population.

Previously published data on acute MI in pregnancy have largely been obtained by literature search and case report review, with cases dating as far back as $1922 .{ }^{1-3}$ Diagnosis and treatment modalities for acute MI have improved since that time.

Women in the study were identified who sustained an acute MI during pregnancy, labor, and delivery, or the 6-week postpartum period and who delivered between January 1, 2010, and December 31, 2013. The remaining non-MI pregnant population was subsequently used as a comparison group for analysis.

The pregnancy complications searched for included preeclampsia, eclampsia, and gestational diabetes as well as identifiable cardiac risk factors, including coronary atherosclerosis, essential hypertension, pregestatioinal diabetes, and disorders of lipid metabolism.

A multivariate analysis was performed to identify risk factors for acute Mi using logistic regression controlling for parity, diabetes, gestational diabetes, chronic hypertension, severe preeclampsia, eclampsia, and maternal age where appropriate.

Eleven Women $(73 \%, \mathrm{P}<.001)$ died from their acute MI and all were diagnosed in the antenatal and intrapartum period. The timing of diagnosis of the acute MI demonstrated equal numbers in the antepartum and postpartum period (Table 1). In Table 1, the demographics of the population are characterized. Women with an acute MI were more likely to be multiparous than those without (Table 1), however, parity was not associated with timing of acute MI.

The maternal age distribution for those with and without an acute MI is demonstrated in Figure 1. Both groups had a normal distribution, with the acute MI group shifted to the older age by approximately 5 years $(\mathrm{P}<.05)$. Maternal age was not associated with timing of acute ML. Six women had multiple gestations $(4 \%)$, and no demographic or timing of acute MI factors were different as compared with singleton women. 
In Table 2, maternal outcomes are separated into timing of diagnosis of acute MI and into those patients who did not have an acute MI. When examining the timing of diagnosis of acute MI, several interesting finding are demonstrated. There were higher rates of maternal and pregnancy complications (prematurity, pregestational diabetes, and mild preecalampsia) associated with the diagnosis of acute MI in the antenatal period as compared with the postpartum period (Tables 2 and 3 ).

Intrapartum diagnosis of acute MI is associated with the highest risk of eclampsia, severe preeclampsia, and maternal mortality and the lowest risk of being diagnosed with coronary artery disease (Table 2), Overall, classic risk factors (coronary artery disease, disorders of lipid metabolism) associated with acute MI were increased in those patients with any acute MI as compared with those without one (Table 2)

Advancing maternal age independently increased the risk of acute MI when all ages were compared with those aged 21-25 years : aged 30-35 years, odds ration (OR) 2.6 (95\% confidence interval [CI] 1.9-6.0); aged 30 years or older, OR 3.4 (2.3-7.9) and chronic hypertension, OR 24.5 (95\% CI 14.8-40.3). Both eclampsia and severe preeclampsia were associated with an increased risk of acute MI : eclampsia, OR 15.3 (95\% CI 5.344.1) and severe preeclampsia, OR 6.9 (95\% CI 5.3-44.1) and severe preeclampsia, OR 6.9 (95\% CI 3.7-13.1).

We may see more pregnant women with an acute MI in the future as more hig-risk women become pregnant. The 3 strongest independent predictors or factors associated with an acute MI were chronic hypertension, diabetes, and advancing maternal age. This older population of pregnant women with preexisting medical conditions may represent a unique group for early diagnosis, treatment, treatment, and further study.)

Besides mortality, there was a significant degree of morbidity with acute MI, which included all types of hypertensive disorders or preganancy, incuding eclampsia, preeclampsia, chronic hypertension, coronary artery disease, and pregestational diabetes. Timing of the acute MI in relation to delivery showed distinct patterns as well. The majority (59\%) of cases of acute MI collected from the literature occurred during the last one third of pregnancy.

Timing of the diagnosis of acute MI in relation to the pregnancy demonstrated interesting characteristics. Women who were diagnosed during the antenatal period had higher mortality and pregnancy complications (Table 2) as compared with having the diagnosis in the postpartum period.

Clearly the antenatal acute MI results in worse pregnancy outcomes when compared to the postpartum diagnosis. The women diagnosed postpartum still had major increases in morbidity (Table2) when compared with women who did not have an acute MI, suggesting the obvious, that any acute MI is a high-risk condition.

Many distinctive characteristics of women with an acute MI were demonstrated. The frequency of women delivering over the age of 40 is only 2-3\%.6 Seven percent of the acute MI patients were older than 40 years and $66 \%$ were older than 30 years. With older women delaying child birth into the fifth decade of life, we need to be more aware of the possibility of acute MI in this population. A most striking finding in patients with an acute MI was the degree of hypertensive comorbidities (Table2). All types of hypertension, either preexisting or pregnancy related, were significantly increased. Clearly, hypertensive disorders must predispose pregnant women who had preeclampsia in the past.7

Both pregestational and gestational diabetes were significantly increased in women with an acute MI, which could be expected due to the fact that diabetes in the general population is associated with an increased risk of acute MI. After correcting for potential confounders, pregestational diabetes was still associated with in increased risk of acute MI, whereas gestational diabetes was not. In our study, coronary atherosclerosis was diagnosed in approximately $28 \%$ of women diagnosed with an acute MI before labor and in $39 \%$ of postpartum patients (Table 2), which is greatly increased over those women without an acute MI.

Obviously, if a woman is diagnosed with an acute MI, some workup will be undertaken to determine a cause for the acute MI, whereas a women without an acute MI would be far less likely to undergo a similar workup.

With more women delaying child birth until the fifth and sixth decades of life, healthcare providers should be aware of the risk factors associated with an acute MI (chronic hypertension, diabetes, eclampsia, and preeclampsia) and potentially perform diagnostic testing on these women in an effort to identify and potentially treat women at high risk of an acute MI. In addition, with many women using assisted reproduction with donated ovum, women aged in their $50 \mathrm{~s}$ and 60 s are regularly becoming pregnant, placing them at an even greater risk of acute MI than at a younger age.

\section{References:}

[1]. Badui E, Enciso R. Acute myocardial infarction during pregnancy and puerperium : a review. Angiology 1996:47:739-56.

[2]. Hankins GD, Wendel GD Jr. Leveno KJ, Stoncham J. Myocardial infarction during pregnancy: a review, Obstet Gynecol 1985:65:139-46.

[3]. Roth A, Elkayam U. Acute myocardial infarction associated with pregnancy. Ann Intern Med 1996:125:751-62.

[4]. Chaithiraphan V, Gowda RM, Khan IA, Reimers CD. Peripartum acute myocardial infarction:management perspective. Am J Ther 2003:10:75-7. 
[5]. Herrchen B, Gould JB, Nesbitt TS. Vital Statistics linked birth/infant death and hospital discharge record linkage for epidemiological studies. Comput Biomed Res 1997:30:290-305.

[6]. Hurst the Heart $12^{\text {th }}$ Edition.

[7]. Braunwald Text Book of Cardiology. 